\title{
The manifestation of intonational focus in Castilian Spanish*
}

\section{Mercedes Cabrera Abreu}

Universidad de Las Palmas de Gran Canaria. Departamento de Filología Moderna

Pérez del Toro, 1. 35003 Las Palmas de Gran Canaria. Spain

mcabrera@dfm.ulpg.es

\section{María Luisa García Lecumberri}

Universidad del País Vasco/EHU. Departamento de Filología Inglesa

Paseo de la Universidad, 5. E-01003 Vitoria-Gasteiz. Spain

fipgalem@vc.ehu.es

\begin{abstract}
In this paper we study the phonological manifestation of various intonational focus domains in Castilian Spanish. We propose that downstep is one of the intonational signallers of focus in this language. The phonological problems of accounting for this phenomenon in Pierrehumbertian models are explored. Alternative solutions are offered following the single-tone model put forward by Cabrera Abreu (2000) within the Government Phonology framework, taking into account other proposals by the author and her co-workers. Typically, the prefocal and focal items participate in licensing relations which integrate them in a well-formed phonological structure in terms of onsets and nuclei respectively as the only two possible constituents within another major constituent, the intonation group. Pitch movements in the prefocal and focal items in the various focus domains are accounted for by the presence / absence of T(one) associated to different constituent boundaries. In addition, the constituent licensing the focal item is preceded by an intonation group whose nuclear constituent is empty, thus showing toneless boundaries which are responsible for the observed downstep effect.
\end{abstract}

Key words: intonational focus, phonological representations, downstep, licensing relations, onset, nucleus, intonation group, $\mathrm{T}$ (one), tone association, constituent boundaries, empty nucleus.

\section{Introduction}

There is general consensus (for instance, Frota 2002 and references therein) that intonational focus can be signalled by various means which include some or all of the following: (a) a particular tonal configuration, (b) some kind of prosodic marking, and (c) a specific phrasal structure. Few studies, however, point at the rather recurrent manifestation of downstep as one of the possible cues speakers resort to in order to signal that a particular item is in focus.

By adopting the analyses we have proposed elsewhere (Cabrera Abreu 1998, Cabrera Abreu et al.1999, Cabrera Abreu 2000), and by offering new data, in this

* The authors wish to thank Timothy Face and Pilar Prieto for their insightful comments on an earlier draft of this article. 
article, we suggest that Castilian Spanish is one of the cases in point. Thus, after studying declarative utterances with the structure SVO, and showing focus on various positions - such as the subject, the verb, the predicate and the object - we consistently observe that, in those cases in which the focused item is non-initial in the utterance (that is, the subject), the peak values are drastically lower than any preceding peaks.

For a set of reasons which we shall review in $\S 3$, a coherent account of such an observation in terms of phonological structure still remains to be put forward. An initial difficulty is found in the account proposed by early Pierrehumbertian approaches to the phenomenon, which rely on the nature of successive pitch accents as the condition for downstep to be interpreted from phonological structure. In terms of such approaches, the trigger for downstep consists of a bitonal pitch accent. This leads to a set of conditions which can hardly be met in the phonological description of many intonation contours. As an alternative, we shall present a solution following the framework proposed in Cabrera Abreu (2000), and which was already put forward in Cabrera Abreu (1998) and Cabrera Abreu et al.(1999), in which the approach to downstep is drastically different. Basically, the trigger is no longer a specific tonal configuration, but rather, it is motivated in phonological representation by the presence of a toneless constituent.

On the way to proposing this view, and offering a complete description of the manifestation of focus in Castilian Spanish, we shall also probe on various issues which have turned out to be controversial, like, for instance, the tonal configuration of the focalised item, and whether or not the right edge of the focalised item is marked by a prosodic boundary as has been suggested for other Romance languages (Estebas Vilaplana 2000, Hualde 2000 (and references therein)). We shall offer our own view also in terms of Cabrera Abreu (2000).

\section{Data collection and description}

The data is from one speaker, and consists in a selection from that found in García Lecumberri (1995), García Lecumberri et al (1997) and Cabrera Abreu et al. (1999). The speaker is female and has a Standard Northern Castilian accent. Utterances were elicited as responses to questions designed to obtain focus on the target positions. Questions were constructed so as to elicit focus for new information rather than contrastive focus. Thus to elicit focus on the subject in the sentence $\underline{e l}$ encarGAdo enviaba los sueldos ('the manager sent the wages') the question posed to the informant was ¿Quién enviaba los sueldos? ('Who sent the wages?'), with narrow but non-contrastive focus on the subject. The speaker answered the questions using sentences which were presented to her in written form but in which there were no indications as to their intonational realization. Scripted material was used to avoid the speaker altering the sentences or eliding old information, which is frequently the case when signalling focus.

The utterances present focus on the subject (initial focus) obtained with the question mentioned above, focus on the verb (medial focus) elicited with the question ¿Qué hacía el encargado con los sueldos? ('What did the manager do with 
the wages?'), focus on the predicate as answer to the question ¿Qué hacía el encargado? ('What did the manager do?'), and focus on the object (final focus) elicited with the question ¿Qué enviaba el encargado? ('What did the manager send?'). As can be seen medial focus is narrow focus on the verb itself excluding the rest of the predicate whereas predicate focus includes under its scope both the verb and the object which follows it.

PitchWorks ${ }^{\circledR}$ is the software used to perform the analysis of the F0 contour. The focal item always shows pitch prominence, and the non-focal items may or may not be accented, as we shall describe below. The utterances were all statements showing the structure subject + verb + object, hence the above mappings between sentence positions and constituents.

\subsection{Initial focus utterances}

In initial focus utterances, focus is normally placed on the subject as illustrated in Figure 1 over the utterance El encarGAdo enviaba los sueldos ("the manager sent the wages'). We use the convention of showing focused items underlined, and accented items in uppercase $)^{1}$.

At the beginning, pitch shows a very soft descending slope over the syllables $\underline{e l}$ encar- going from $193 \mathrm{~Hz}$ to $166 \mathrm{~Hz}$. There is a small valley $(166 \mathrm{~Hz})$ corresponding to the effects of micro-intonation of the voiced velar fricative [y] of the accented syllable $-G A$ - . After this point pitch starts to rise abruptly, reaching a peak of $243 \mathrm{~Hz}$ over the vocalic element of the syllable $-G A-$. Next, the F0 descends rapidly over -do-, being the syllable en-from enviaba the lowest point at $165 \mathrm{~Hz}$. Finally, the F0

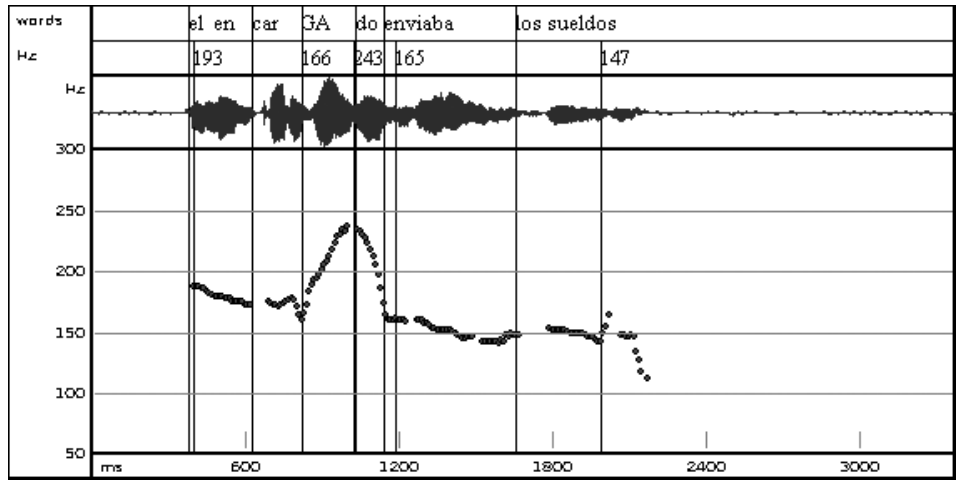

Figure 1. Waveform and F0 contour of the utterance El encarGAdo enviaba los sueldos (initial focus).

1. The F0 contour is a reproduction from one of the authors. 
contour descends gradually until the end over the syllables -viaba los sueldos at $147 \mathrm{~Hz}$.

\subsection{Medial focus utterances}

Figure 2 illustrates the same utterance as before, but this time, with focus placed on the verb, enviAba.

The F0 contour begins with a short and gradient falling movement (from 183 $\mathrm{Hz}$ to $163 \mathrm{~Hz}$ ) over el en-. After this point pitch starts rising over-carGA-, reaching a peak $(209 \mathrm{~Hz})$ at the syllable -do- of encarGAdo. As can be observed, the focal verb shows a falling movement over the first syllable, en-, and the lowest point in this valley is manifested over the syllable $-v i$ - which also participates in another rising movement together with the syllable $-A-$, whose peak is located at $183 \mathrm{~Hz}$. The next falling movement is initiated over this same syllable, and continues to descend over the last syllable, of enviAba, $-b a(147 \mathrm{~Hz})$; the lowest point $(131 \mathrm{~Hz})$ of this third valley occurs at the word los. The F0 contour now shows two discontinuities, - due to the fricative sounds in los and suel- - which are followed by a small jump up to $147 \mathrm{~Hz}$ for the vocalic sounds in suel-. Finally, there is a another short falling movement over sueldos.

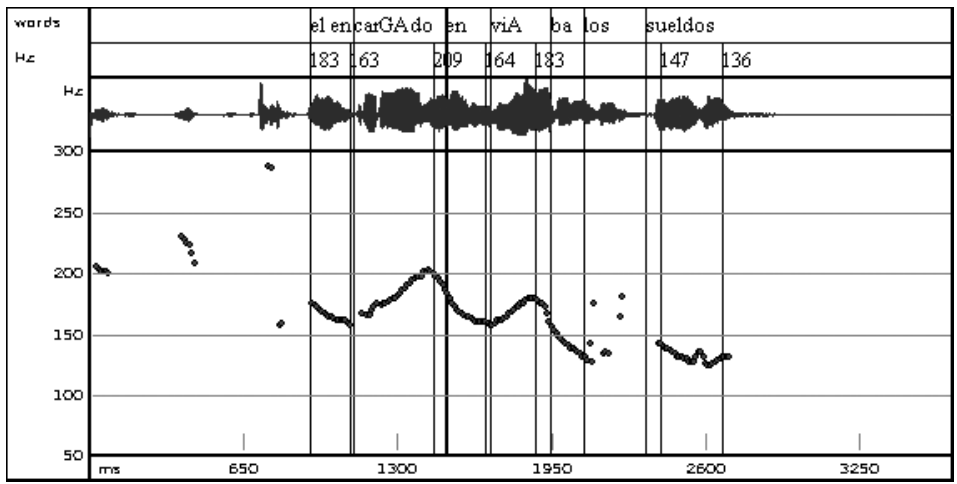

Figure 2. Waveform and F0 contour of the utterance El encarGAdo enviAba los sueldos (medial focus).

\subsection{Focus on the predicate}

Predicate focus utterances are of the type illustrated in Figure 3.

This time focus is placed over enviAba los sueldos. At the beginning, the F0 contour is relatively low over el encar- $(170 \mathrm{~Hz})$, and then starts rising over -GA$(197 \mathrm{~Hz})$. The highest point is reached in $-d o(222 \mathrm{~Hz})$. There is now a falling movement over envi-, to $180 \mathrm{~Hz}$, which is followed by another rise. The second 


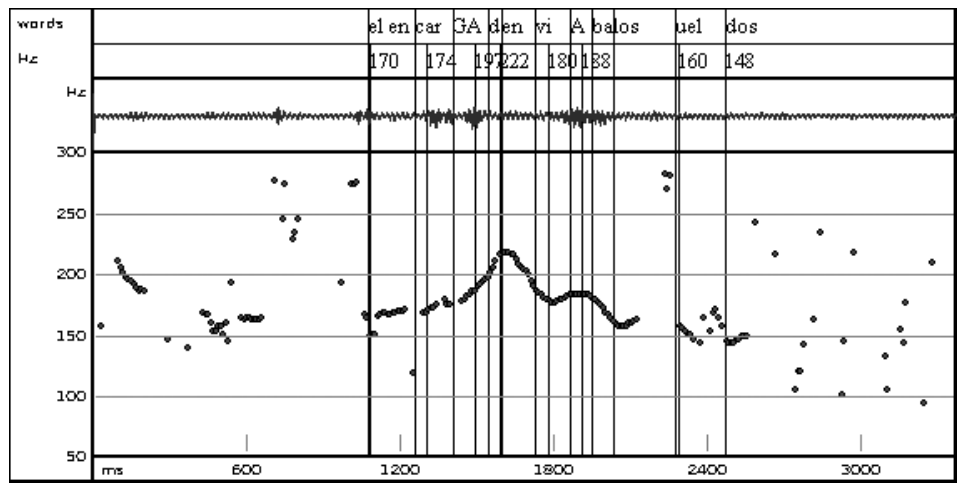

Figure 3. Waveform and F0 contour of the utterance El encarGAdo enviAba los sueldos (focus on the predicate).

peak $(188 \mathrm{~Hz})$, which is slightly lower than the first, corresponds to the syllable $\underline{A-}$ of enviAba. After that, pitch drops gradually over $\underline{-b a}$, and upto -los $(162 \mathrm{~Hz})$. Finally, the signal is rather dispersed, but some relevant points can be clearly observed. There is a gentle gradient from los suel- $(160 \mathrm{~Hz})$ to $-\underline{d o s}(148 \mathrm{~Hz})$.

\subsection{Final focus utterances}

As shown in Figure 4 below, the F0 contour begins by showing a plateau over $\mathrm{el}$ encar-, and then rises over-GAdo and reaches a peak $(202 \mathrm{~Hz})$ shared by the end of $-O$ - in encarGAdo, and the beginning of the vowel $e$ - in enviAba. This gradual rising movement is repeated over the syllables $-A b a$ and ends in a peak at $208 \mathrm{~Hz}$

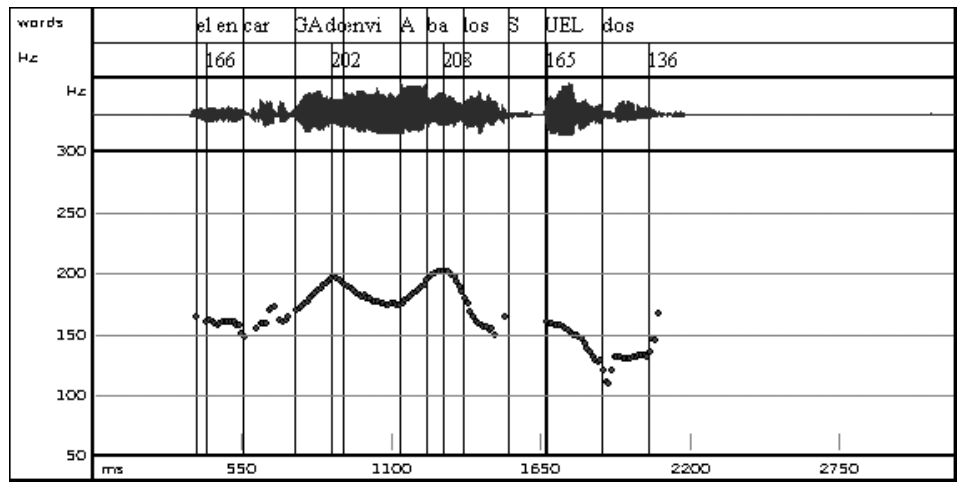

Figure 4. Waveform and F0 contour of the utterance El encarGAdo enviAba los $\underline{\text { SUELdos }}$ (final focus). 
(notice that this second peak is higher than the first one). The F0 contour joining these two rising movements shows a slope over the syllable -vi. After this peak, there is a descending movement which starts at the end of the last vowel $-a$ in enviAba, and reaches its lowest point over the fricative sound at the end of los and at the beginning of $\underline{S U E L d o s^{2}}$. Then, the F0 shows a gap which corresponds to the fact that the fricative sound is partially devoiced. After that, the contour starts again at $165 \mathrm{~Hz}$ and falls over $-U E L$-. The lowest point of the F0 contour is found at 136 $\mathrm{Hz}$ over the syllable - dos.

\section{Discussion and observations}

\subsection{Initial focus utterances}

In terms of AM theory, the main issue here is whether the rising movement over $-G A$ - is part of a bitonal pitch accent of the shape $\mathrm{L}+\mathrm{H}$ as proposed by Face (2002) and Hualde (2000) — and if so, which of the two tones is associated with the starred syllable - , or whether such a movement is an interpolation between an initial default $\mathrm{L} \%$ and $\mathrm{H}^{* 3}$.

In relation to which of the two tones counts as the starred tone of the bitonal pitch accent, Face (2002) proposes $\mathrm{L}+\mathrm{H}^{*}$, whereas Hualde proposes $(\mathrm{L}+\mathrm{H})^{*}$. Face suggests that this pitch accent $\mathrm{L}+\mathrm{H}^{*}$, is the one used for items showing a rising movement standing in nuclear position, and in narrow focus. By contrast, nonnuclear accents show this other configuration: $\mathrm{L}^{*}+\mathrm{H}$. As a counter argument, Hualde claims that nuclear and non-nuclear rising accents are non-contrastive in Spanish, and that the difference in alignment of the rising movements arises as a consequence of the fact that in nuclear position they are followed by L- whereas in prenuclear position they are not. That is, L- 'pushes-in' the rising movement, so that it takes place earlier in the accented syllable, and therefore, is not delayed until de post-tonic one. In addition, he argues that the best representation which captures the characteristic feature of an abrupt rise in pitch across the stressed syllable is $(\mathrm{L}+\mathrm{H})^{*}$, with both tones associated to the stressed syllable, hence his use of parenthesis.

Given this context, we claim the following:

1. According to our data analysis, we agree with Face (2002) in that nuclear and pre-nuclear rises sound radically different.

2. We also support his proposal in the sense that the contrasting effect results from the tonal configurations, and not necessarily from the presence of the L- phrase accent after the nuclear accent ${ }^{4}$.

3. Hualde's approach in which $(\mathrm{L}+\mathrm{H})^{*}$ stands as the best descriptively adequate representation for nuclear rises may lead onto the issue about whether or not

2. See discussion about whether or not sueldos shows pitch prominence in $\$ 3.4$.

3. Prieto (1999) and Nibert (2000) defend $\mathrm{H}^{*}$ as the pitch accent which accounts for rises in Spanish.

4. As we shall see below in $\S 4.3$ and $\S 5.1$, the fact that the phrase accent (or a prosodic-edge marker in terms of Cabrera Abreu (2000)) appears after the focal item is motivated theory internally. 
there is a contrast between such a tonal configuration and $\mathrm{L}+\mathrm{H}^{*}$. In other words, by assuming, on the one hand, that two tones can be associated to a stressed syllable, and on the other, that the sequence of a trailing tone and a starred tone are permitted in the model, we may conclude that such configurations stand a chance of being contrastive. This is something still to be attested.

4. We are on a similar line to Prieto (1995), García Lecumberri et al. (1997), and Nibert (2000) in the sense that we suggest that the nuclear pitch accent shows a characteristic falling pitch movement, which is accounted for by a specific tonal association, and that the rising movement before it arises as a consequence of interpolation. In addition, although this has to be confirmed with further research, we assume that the greater the degree of focus a given speaker assigns to an item, the sharper and the earlier the rising movement over such an item, as this arises as a consequence of compensating for the characteristic falling movement which will follow 5 .

5. In addition, rising pre-nuclear patterns are accounted for by a different phonological structure. We shall turn to this below in §5.2.

In relation to the $\mathrm{F} 0$ shape of the initial focus utterances, it must be pointed out that Face (2002) describes another F0 shape for Madrid Spanish different from the one we have come across for Castilian Spanish in our data. Some of Face's contours show a rising movement over the focal material followed by relatively high pitch at the end of the focused word. He accounts for such ending by means of H-. These contours stand in sharp contrast to ours, which constantly show a relatively low ending point after the focal word, which could be accounted for in terms of AM theory as L-. These differences may be due to the fact that our speaker was born in the area surrounding Burgos, a town which can be treated as belonging to a type of Standard Northern Castilian accent, and currently lives in Vitoria (the south of the Basque Country), whereas Face's speakers are circumscribed to Madrid, and thus, may possibly be displaying a different intonational accent.

\subsection{Medial focus utterances}

As we have already stated in the previous section, there are conflicting views within AM theory in relation to the description of rising movements in Castilian Spanish. Notice that, as pointed out also by Hualde (2000), the rising movement takes place clearly over-GA-, and for this reason he justifiably rejects other proposals, adopting the representation with parenthesis $(\mathrm{L}+\mathrm{H})^{*}$ for all rising movements, regardless of their position in the utterance. However, we shall adopt an alternative view on two counts. First, the rising movement in pre-nuclear position is accounted for by a phonological representation different from nuclear position, and second, the

5. This can be easily observed in our own emphatic production of a high fall movement over a single syllable. The more the emphasis, the more the effort, and the longer the rising movement before the subsequent fall. 
characteristic downstep effect ${ }^{6}$ between the pre-nuclear and focal nuclear pitch prominences arise as a consequence of phonological structure. For notice that the height of the peak in the pre-focal material $(209 \mathrm{~Hz})$ is certainly higher than the second peak in the focal material $(183 \mathrm{~Hz})$. This is further supported by the fact that, in those cases in which the second peak does not coincide with the focal material (like in the case of focus on the object) such second peak does not show downstep (first peak, $202 \mathrm{~Hz}$; second peak, $208 \mathrm{~Hz}$ ).

In relation to the focal material, the accented syllable is nearer the peak than to the actual rising movement. In fact, most of the vowel duration takes place over the peak. This being observed, we cannot follow Hualde's (2000) proposal $(\mathrm{L}+\mathrm{H})^{*}$, since it is descriptively inadequate. Clearly, the accented nuclear syllable must be something like $\mathrm{H}^{*}$, as we already defended in García Lecumberri, et al. (1997) for these type of utterances as well.

Once we have assumed that the focal material is accounted for by $\mathrm{H}^{*}$, we must consider another feature observed in this type of utterances, and which we pointed out in García Lecumberri et al. (1997). Such a feature consists in the observed fact that the focal accent is downstepped. The difficulty encountered then was that the early proposals offered in terms of AM theory disallowed us to show that $\mathrm{H}^{*}$ suffered downstep with respect to the previous tonal prominence. In other words, the trigger, $\mathrm{L}^{*}+\mathrm{H}$, and the target, $\mathrm{H}^{*}$, were separated by a phrase accent, $\mathrm{H}-$, and in this situation, the theory did not predict the downstep effect to take place. Thus, it was observed that the way downstep was encoded in Pierrhumbertian early analyses lead onto unsolved difficulties. Alternatively, we suggested that if downstep were treated as an independent choice, unrelated to the specification of a preceding pitch accent as being bitonal (as proposed also by the ToBI followers ${ }^{7}$ ), then much of the difficulty would disappear. In a later work, Cabrera Abreu (1998), following the framework set by Government Phonology, claims that such an item is a toneless constituent which is integrated into phonological structure as an empty nucleus. We shall turn to this later in $§ 4.4$.

\subsection{Focus on the predicate}

As already reported in García Lecumberri and Cabrera Abreu (1999), there is a strong similarity between the F0 contour for this type of utterances, and the ones showing focus on the verb in the sense that, they both show pitch prominence in the verb item, and after that, the F0 contour shows a falling movement. That is, they expected to find two pitch prominences when the focus was placed over the whole predicate, - one in the verb and another in the object. Instead, they reported a subtle difference in the falling movement of the F0 contour after the peak in the verb item, which lead them to conclude that these two types of utterances are ambiguous.

6. Future studies will include detailed research into declarative utterances in order to test claims which point to the fact that such utterances also show downstep even though they lack a focal item.

7. See Ladd (1996). 


\subsection{Final focus utterances}

The pre-focal material in this type of utterances shows a sequence of rising movements over the subject and the verb similar to the one we observed over the prefocal material in utterances with medial focus. The last peak $(165 \mathrm{~Hz})$ on the focal material is downstepped with respect to the preceding peak $(208 \mathrm{~Hz})$. Finally, notice that the focal material shows a clear falling movement over the accented syllable SUELdos. Thus, this can be taken as further evidence of the fact that the focal accent is accounted for by $\mathrm{H}^{*}$ and that the rising movement over the pre-tonic syllables (if there are any) stands as a consequence of a phonetic effect, and that it is not motivated by the bitonal nature of a rising pitch accent.

It must be admitted that, occasionally, our speaker was inconsistent about the intonation contour of the utterance bearing focus on the object. In fact, when analysing our data, we found some difficulty in identifying the last pitch prominence. Comparing our perception of this last prominence over SUELdos with the curve over the same word - which is de-accented in medial focus - we found that, when analysed in isolation, the same item sounds very similar whether or not it is focalised ${ }^{8}$. However, when analysed in its own context, there seemed to be some kind of difference. This led us to assume that, indeed, $\underline{S U E L d o s}$ is pitch prominent, and that there are other factors at work, such as the pre-focal contour, and the downstep effect.

Summing up the description of what we have observed in utterances showing intonational focus, we must bear in mind the following points in order to put forward a descriptively and explanatory adequate phonological account:

1. The nature of pitch accents in pre-focal and focal material is different. The former are rising movements, whereas the latter is characteristically a falling movement followed by a prosodic edge marker.

2. The observed rising portion over the pre-tonic syllables in initial focus may be the result of a phonetic interpolation.

3. The focal item in non-initial position is marked by the downstep effect.

The phonological account will be based on the model proposed by Cabrera Abreu (2000). Therefore, in the next section we present an outline of the main ideas of that model.

\section{Cabrera Abreu (2000)}

Some of the main tenets presented in Cabrera Abreu (2000) include the following:

1. A single tonal unit $\mathrm{T}$;

2. The association of $\mathrm{T}$ to the boundaries of domains or constituents;

3. The assumption that metrical structure feeds intonational phonology, which follows from pragmatic principles;

8. As pointed out by our reviewer, the last pitch accent can be affected by a process of final lowering to the extent that the rising movement in the F0 contour can be absent. See Prieto (1998). 
4. The formalisation of constituents in intonational phonology in terms of licensing principles as the nucleus, the onset, and the intonation group;

5. The integration of empty categories into phonological structure as empty nuclei and their role in the account of downstep.

\subsection{A single tonal unit $T$ and its association to the boundaries of constituents}

Current models of intonation within the AM Theory assume that there are two tonal units in phonological structure: H(igh) and L(ow). Such models contrast sharply with the one we are concerned with here, which is fully embedded in the framework of Government Phonology (Kaye, Lowenstamm and Vergnaud (1990), and Harris (1994)), and which proposes a single T(one) only, which corresponds to former $\mathrm{H}$. In addition, $\mathrm{T}$ can only be associated to boundaries of constituents, rather than to both boundaries and accented syllables, as was formerly customary.

Given this, relatively high pitch is accounted for by the association of tone to a boundary, and consequently, relatively low pitch is accounted for by a toneless boundary9. We illustrate this in (1) below, where we show a preliminary phonological structure which accounts for an intonation domain (shown by means of the outermost boundaries) which shows a relatively high pitch constituent (T associated to the first pair of inner boundaries) followed by a falling pitch constituent ( $\mathrm{T}$ associated to the left boundary of the second set of inner boundaries).

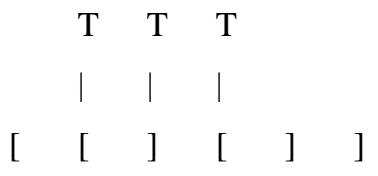

\subsection{Metrical structure versus intonational phonology}

The idea about constituents originates from the recurrent concepts conveyed by the terms 'Head' and 'Nucleus', and with that of the 'Word Group' in the British tradition (O'Connor and Arnold 1973) of intonation models. They count as the skeletal structure of intonation which Cabrera Abreu (2000) informally shows as follows:

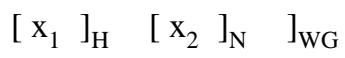

The $x \mathrm{~s}$ in (2) correspond to the onset syllable $\left(\mathrm{x}_{1}\right)$ and the nuclear syllable $\left(\mathrm{x}_{2}\right)$ in O'Connor and Arnold's terms. The Head $(\mathrm{H})$ domain and the Nucleus $(\mathrm{N})^{10}$ domain are integrated into the Word group (WG) (or also referred to as the Intonation Group) ${ }^{11}$.

9. In her work, Cabrera Abreu (2000) offers a detailed description about the phonetic interpretation of toneless boundaries, and uses the term Gravitation Effect to refer to a down-pushing force on the $\mathrm{F} 0$ in those cases in which boundaries remain toneless.

10. These terms are later formalised as the 'onset' and the 'nucleus' respectively.

11. This skeletal structure of intonation constituents is formalised below in $\S 4.3$. 
Next, the intonational structure represented in (2) is mapped straightforwardly with the metrical structure, and more precisely with the level relevant for intonational computations. Cabrera Abreu (2000) takes for granted that such a level shows two positions, as illustrated below in (3) ${ }^{12}$ for the utterance anOther Orange.

\begin{tabular}{|c|c|c|c|c|c|}
\hline & $\mathrm{T} \quad \mathrm{T}$ & & $\mathrm{T}$ & & \\
\hline & 1 1 & & $\mid$ & & \\
\hline [ & 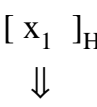 & & $\begin{array}{r}{\left[x_{2}\right.} \\
\Downarrow\end{array}$ & ]$_{N}$ & $\mathrm{~J}_{\mathrm{WG}}$ \\
\hline & $\mathrm{x}$ & & $\mathrm{x}$ & & level 1 \\
\hline X & $\mathrm{x}$ & $\mathrm{x}$ & $\mathrm{x}$ & $\mathrm{x}$ & level 0 \\
\hline an & $\mathrm{O}$ & ther & $\mathrm{O}$ & range & \\
\hline
\end{tabular}

Another stage in the presentation of the intonation structure is to assign tone to the intonation boundaries in order to illustrate the pitch movements we showed in (1), bearing in mind that tone is assigned to the boundaries offered by intonational phonology ${ }^{13}$, that is, those corresponding to the Head domain, the Nucleus domain, and the Word Group. Of course, such boundaries subsume all the boundaries in metrical structure (which are not illustrated in (3), but which we have shown in (4)), and all the positions therein.

(4)

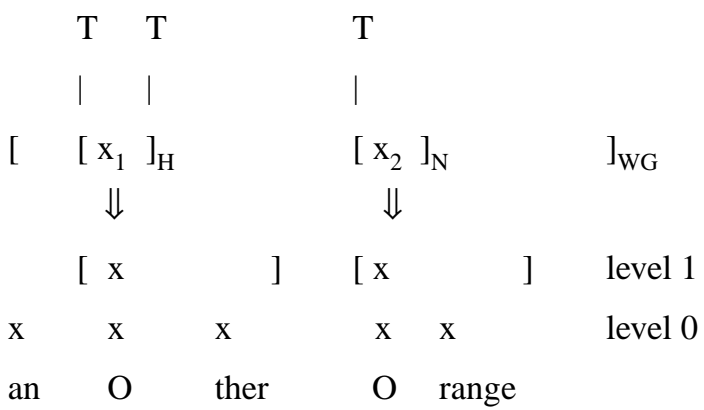

12. See Cabrera Abreu (2000) for further details about metrical structure. Also, there she draws a parallel between this situation and the one found in syllable structure in Government Phonology, where the topmost node, the syllable node, is irrelevant for phonological computations, whereas the lower two nodes, the onset node, and the nucleus node, do show a theoretical status.

13. Licensing principles also operate between constituent boundaries. We shall not refer to them in any detail here since they are irrelevant for the present paper. The only idea the reader needs to know about is that the rightmost boundary of the nucleus and leftmost boundary of the intonation group cannot bear T (see the Licensing Inheritance Principle in Cabrera Abreu (2000) for further details on this topic). 
The structure in (4) is understood as follows: a tonal specification associated to the intonational boundaries has an effect on $\mathrm{x}_{1}$ and $\mathrm{x}_{2}$, and all the syllables (or positions) 'below' (level 1 and level 0 ) as indicated by the metrical structure. For instance, $\mathrm{T}$ associated to the leftmost and rightmost boundaries of the Head, and to the leftmost boundary of the Nucleus specify tonal characteristics (interpreted as relatively high pitch over -nother followed by a falling pitch movement over Orange, as we already stated above). Finally, the pitch interpretation of the syllable $a$ - from another is read-off from the tonal value specified at the leftmost boundary of the Intonation Group.

\subsection{Justification and consequent formalisation of constituents in intonational phonology}

In her chapter 4, Cabrera Abreu (2000) proposes that the intonational manifestation of focus, following pragmatic principles, is the factor which provides evidence for the existence of two independent constituents, the onset and nucleus. In this sense, old or given information is treated as part of the onset, whereas the focal item (which bears the nuclear accent) belongs to the nucleus. Consequently, in the case of intonational focus in Castilian Spanish which we shall turn to below, we expect to find the focal item as part of a nuclear constituent, and the pre-focal items as part of an onset constituent ${ }^{14}$. Thus, the obligatory syllable (the nuclear syllable), is aligned with the rightmost edge of the intonation domain, and the optional syllable (the onset syllable), is aligned with the leftmost edge of the intonation domain.

Given this, a principle of domain formation that is pragmatically determined is stated in the following terms:

(5) (a) the nuclear domain (determined by the nuclear syllable) is aligned with the rightmost edge of the intonation domain;

(b) the onset domain (if present) is aligned with the leftmost edge of the intonation domain.

An idea shared by several phonological frameworks (Government Phonology, Dependency Phonology, etc.) is that the obligatory presence of a constituent in the structure counts as an indication of its headship. Thus, in the specific case we are concerned with here, the fact that the nucleus is the only obligatory constituent in intonation is reason enough for classifying it as the dominant (or licensor or head) constituent and, consequently, the onset as the dominated (or licensee or complement) constituent. In addition, it should be noted that implicit in the identification of the licensor/licensee dichotomy between constituents is the claim that these two domains stand in a close relation. These two ideas are formalised in Government Phonology under the principle of onset licensing, which is stated as follows:

14. But see the situation found in the case of focus placed on the object, and how some of the prefocal material is not sanctioned by an onset, but instead by a nucleus. 
(6) Onset licensing: an onset head position must be licensed by a nuclear position.

Let us now illustrate the principle in (6) by analysing the utterance in (4) and repeated below in (4'). The phonological representation shows the nuclear constituent and the onset constituent; the asymmetric relation between them is encoded by means of an arrow (the outer boundaries correspond to those of the intonation group):

$\left(4^{\prime}\right)^{15}$

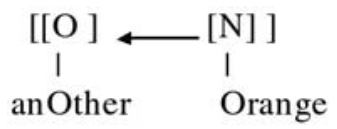

The head position in the nuclear constituent ( $O$ - from Orange) licenses the onset head position ( $-O$ - from anOther). The directionality of licensing between these constituents shown by the pointed arrow goes from right to left, as an indication that the rightmost constituent is the licensor. Thus, this representation already conforms to the principle stated in (6). $a$ - in anOther, which corresponds to O'Connor and Arnold's Pre-Head, is not incorporated into an independent constituent, since no complementary assigned constituent is assumed in the present restricted approach. Instead, $a$ - is left as part of the intonation group.

In the paragraphs above, we have reproduced the evidence which leads Cabrera Abreu (2000) to propose two smaller constituents in intonation, the nucleus and the onset, but as she claims, an intonation group may show a nuclear constituent only, since this is the obligatory constituent (whilst the onset is optional). Similarly, an intonation group which shows a lone onset constituent fails to be generated by the grammar, since this hypothetical structure violates the principle in (6): such an onset would not be licensed by a nuclear position.

Another structure which is ruled out by the principle of onset licensing is a sequence of two nuclei in a single intonation group. At first glance, the principle in (6) predicts a licensing relation between constituents of different ranks (i.e. an onset must be licensed by a nucleus) and not between constituents of equal rank, thereby ruling out a sequence of two onsets or of two nuclei ${ }^{16}$.

15. Lines and arrows represent licensing paths.

16. In Cabrera Abreu (2000) this claim is loosened on the grounds that one of such nuclei is empty. However, this may eventually prove to be too powerful and lead to undesirable phonological structures in which the presence of empty nuclei is unrestricted. Therefore, the conditions for the distribution of empty nuclei require further research. In the present paper, we shall not adopt this idea for the reasons stated in $\$ 4.4$ below. 


\subsection{The integration of empty categories into phonological structure and their role in the account of downstep}

Given the idea presented above in $\S 4.1$ that toneless boundaries constitute the only structural factor which can account for the phonetic result of pulling the F0 downwards, it follows that they are also involved in the manifestation of the downstep effect. Indeed, Cabrera Abreu (2000) proposes that there are some domains in phonological representation which lack any melodic content, and whose boundaries do not constitute potential landing sites for tone. Such empty domains have the effect of triggering downstep of a $\mathrm{T}$ which is associated to a following domain ${ }^{17}$. For instance, (7) below illustrates a structure which is interpreted as two falling movements over anOther and Orange, only that the second one starts at a considerably lower level than the first one. The fact that the second $\mathrm{T}$ is preceded by an empty domain is read-off in the phonetic manifestation as an indication that its value is lower than that of the preceding $\mathrm{T}$.

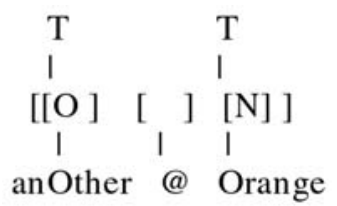

The symbol '@'18 in (7) is used — following the tradition set by Government Phonology - as an informal device to indicate that the domain which acts as its licensor is empty of any melodic content.

The next step in Cabrera Abreu's model is to identify the nature of the empty domain as part of the constituent structure, whether such a constituent is part of an onset or of a nucleus. After showing some evidence from segmental phenomena, she adopts in intonation the analysis put forward in Government Phonology for syllable structure, in which specific nuclei can remain empty. In this sense, the structure in (7) above is reproduced in (8) below:

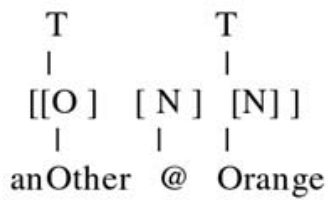

17. See Cabrera Abreu (2000: 121-122) for a detailed description of the phonetic interpretation of empty domains in terms of Lincensing Inheritance.

18. Also see Cabrera Abreu (2000:112) for her view on how ' @' is encoded in the linguistic message following pragmatic principles. 
@ is licensed in phonological structure by a nucleus to which we shall refer as being empty since it is devoid of any melodic content.

In the same way as an onset and a nucleus are integrated into phonological structure by means of licensing relations, the presence of an empty nucleus, being a nucleus, is also sanctioned by the licensing relations stated below:

(9) Inter-nuclear licensing: $\mathrm{N}$ licenses other Ns (which are empty) (Cabrera Abreu 2000: 118).

Thus, the structure in $(8)^{19}$ shows the following licensing relations:

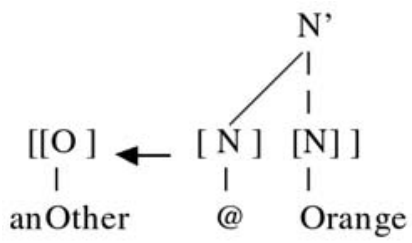

By onset licensing (see (6) above), the onset head position, $-O$ - is licensed by the neighbouring nucleus, which happens to be empty. Furthermore, by inter-nuclear licensing such nucleus is itself licensed by the projection of the filled nucleus. As can be seen in the structure above, Cabrera Abreu also resorts to showing dependency relations among constituents by means of projecting a given item to higher levels in the structure. Now that all constituents are integrated into a well-formed phonological structure, this can be phonetically interpreted following the tonal specifications shown above in (8).

Unfortunately, however, as already pointed out by Cabrera Abreu (2000:115) herself in a footnote, the introduction of 'levels of projection in the representation may lead us to assume the existence of extra domains, and consequently, of extra boundaries as potential landing sites for T'. Since this may lead on to further problems of overgeneration and indeterminacy, she disregards such potential domains as markers of intonational phenomena, but she still adopts such a representation.

Although we believe the decision not to propose further domains to be the right one, it seems that in her discussion and analysis of contours showing downstep in English, the author missed the opportunity to take advantage of developing the proposal she put forward in her chapter 2 (p. 40-41), later formalised in her chapter 4 (p. 60-64), and which we have presented above in $\$ 4.2$ and $\$ 4.3$. Recall that there she offers convincing justification for assuming a binary relation of constituents in intonational phonology, in the sense that she defends the view that a

19. We have excluded the tonal specifications in this structure for the purposes of clarity. Notice also that this representation is not adopted by the authors of this paper to account for the manifestation of accentual focus in Spanish. See argumentation in the following paragraphs. 
well-formed intonation group maximally shows an obligatory nuclear constituent, and an optional onset constituent. Presumably, had she adopted this idea to its ultimate consequences, some of the phonological structures she proposes to account for the case of downstep in English, included in chapter 6 (109:141), would have had to be revised.

\section{The analysis of focus}

In two later papers - but which were published earlier than her book - (Cabrera Abreu (1998) and Cabrera Abreu et al.(1999) Cabrera and her colleagues, who are aware of the benefits implicit in her view of a maximally binary constituent structure in intonational phonology, analyse the manifestation of accentual focus in English and Spanish in these terms, keeping the idea that ' @' is licensed by a nucleus, and incorporating other proposals already included in Cabrera Abreu (2000). Let us turn to an example reproduced from Cabrera Abreu et al.(1999) illustrating all this in the following paragraph.

$(11)^{20}$

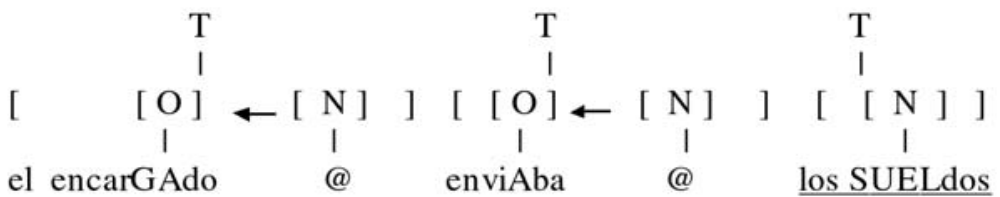

The phonological structure shown above corresponds to the utterance $e l$ encarGAdo enviAba los SUELdos in which the object los SUELdos is in focus. There are three intonation groups, of which the first two are maximally binary, and the third one (responsible for the focal item) shows a single nucleus. Notice that, in the former intonation groups, each empty nuclei licenses an onset. The question which remains unsolved in this discussion is how such empty nuclei are themselves licensed. Although not mentioned explicitly in their paper, Cabrera Abreu, García Lecumberri, Maidment and Takahashi implicitly resort to Cabrera Abreu's (2000) Final-empty nucleus parameter ${ }^{21}$, whereby a final nucleus may be licensed to remain empty, so that the fact that they stand at the right edge of the intonation group sanctions their presence in phonological structure.

The account of downstep in terms of an empty nucleus in phonological structure counts as an advantage over the proposal put forward in early Pierrehumbertian works, and also offers a rather different view from the one defended in AM theo-

20. In our account of final focus utterances in $\$ 5.4$ below, we shall revise this structure since we defend the view that it is descriptively inadequate.

21. This proposal is also adopted from the account of English final consonants in terms of Government Phonology (Harris 1994). 
ries, all of which we mentioned in $\$ 3.2$ above. For one, the fact that the presence of a constituent (an empty nucleus) is responsible for the phenomenon of downstep in the F0 signal suggests that its occurrence is not directly linked to a particular sequence of pitch accents, in which the conditions that the trigger must be bitonal, and that it has to stand next to the target must be met. For the other, it supports the idea that the nature of downstep is unrelated to any particular tonal specification, but rather to the organisation of constituents in phonological structure.

Let us now turn to the phonetic interpretation of the structure in (11). The first two toneless boundaries and $\mathrm{T}$ associated to the right boundary of the first onset account for the relatively low F0 contour over el encarGA-, the rising movement over $-G A$-, and the peak in -do and $e n-$. The empty nucleus and the following toneless boundaries are responsible for a slight drop and short plateau over $-v i A-$. T associated to the right boundary of the second onset accounts for another rise to $-b a$. The second empty nucleus and the toneless right boundary of the second intonation group, and the left one of the third intonation group are all in charge of a drop in pitch over $\underline{l o s}$. Finally, $\mathrm{T}$ associated to the left boundary of the third nucleus and the remaining toneless boundaries are responsible for the rising movement over $\underline{S U E L-}$ and subsequent falling movement to - dos.

In this paper, we adopt this latter proposal in which there is no projection of the nucleus for two reasons: first, as we stated above, it is convincingly defended in Cabrera Abreu (2000), and second, as reported in the two pieces of work mentioned in the previous paragraphs, it nicely accounts for the behaviour of accentual focus in Spanish, which is the intonational phenomenon we are concerned with here $^{22}$. In this context then, the phonological instructions we need to follow in order to account for the data under study are the following:

(12) (a) the nucleus is the only obligatory constituent in an intonation domain;

(b) if there is an onset, then it must be licensed by a nucleus by onset licensing.

(c) Final empty nucleus parameter ON/OFF.

\subsection{Initial focus}

The phonological representation which accounts for initial focus utterances is as follows:

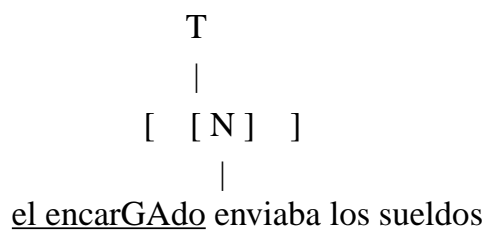

22. It remains for future research to develop the revision of the structure proposed in (10) above, since it is out of the scope of this paper, as here we are concerned with intonational focus in Spanish, and not downstep in English. 
The focal item el encarGAdo is licensed by a nucleus which is the obligatory constituent in an intonation group, and is aligned with its rightmost edge. Notice that such an alignment of the nucleus to the right edge of the intonation group can be assumed to be an equivalent of what was reported by some of the AM theory followers (and which we mentioned in $\$ 3.1$ above) in relation to the presence of L- as a marker of the focal item.

The left intonation group boundary (interpreted in terms of Gravitation Effect or as a down pushing force being exercised on the F0 (see note 6 above)) together with $\mathrm{T}$ associated to the left boundary of the nucleus are responsible for the rising movement over $-G A$ - . Then, the falling movement observed over $-d o-$, and the following relatively low pitch over enviaba los sueldos is accounted for also by the presence of $\mathrm{T}$ associated to the left boundary of the nucleus, and the remaining toneless boundaries.

In view of the structure in (13), and in relation to our discussion in §3.1, we are now in a position to claim that the F0 shape over el encarGA- arises as a consequence of interpolation, and not of a specific tonal configuration including $\mathrm{L}$. Of course, this follows from the fact that in the model adopted here, $\mathrm{L}$ is non-existent. In addition to this, we further claim that any discussion in relation to which tone of the sequence $\mathrm{L}+\mathrm{H}$ counts as the starred tone is a matter of phonetic detail which stands outside phonological representation. Another issue we introduced in $\$ 3.1$ and $\S 3.2$ had to do with the account of rising movements in nuclear (or focal) and prenuclear pitch accents. Notice that the former is accounted for here by the fact that the leftmost boundary of the intonation group is toneless, and also by the association of $\mathrm{T}$ to the leftmost boundary of the nuclear constituent ${ }^{23}$. In the following section we shall turn to a description of the latter.

\subsection{Medial focus}

Following Cabrera Abreu et al.(1999), and the theoretical points we introduced in the previous sections, the phonological structure responsible for medial focus utterances is illustrated in (14):

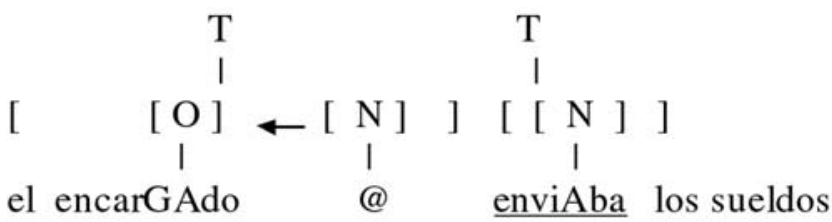

The focal constituent enviAba, being the obligatory one, is licensed by a nucleus. $T$ associated to its leftmost boundary is responsible for the peak on $-\underline{A-}$. In

23. Recall that in Cabrera Abreu's model, T cannot be associated to the right boundary of the nucleus due to the directionality of licensing at the level of boundaries. 
assigning constituent structure to the rest of the representation, our reasoning proceeds in the following way: by $(5 b)$ the prefocal item is licensed by an onset, and by (6) the onset must be licensed by a nucleus. Given that we observe the downstep effect in the signal, and that an onset must be licensed by a nucleus, we assume that there is an empty nucleus in the structure, who is responsible for licensing the onset. By (12c) the empty nucleus is licensed by the fact that it is aligned with the right edge of an intonation group. Its phonological interpretation is manifested in terms of the value of the following $\mathrm{T}$, which is pulled down in F0, so that its pitch value is never as high as the preceding $\mathrm{T}$.

According to the structure in (14), the alleged rising movements observed in Spanish and which we described in $\$ 3.1$ and 3.2 are drastically different. The prefocal movement is accounted for by $\mathrm{T}$ associated to the right boundary of the onset constituent, whereas the focal one is described in terms of $\mathrm{T}$ associated to the left boundary of the nucleus. Thus, the differences in the F0 shape arise as a consequence of tonal association to different constituents and also to the left or the right boundary in each case.

\subsection{Focus on the predicate}

As already stated in §3.3, García Lecumberri and Cabrera Abreu (1999) reported an ambiguity between this type of utterances and the ones showing focus on the verb. This is further confirmed by the phonological structure we propose for utterances with focus on the predicate, which show the same constituent structure as the one shown in (14), and which we illustrate in (15).

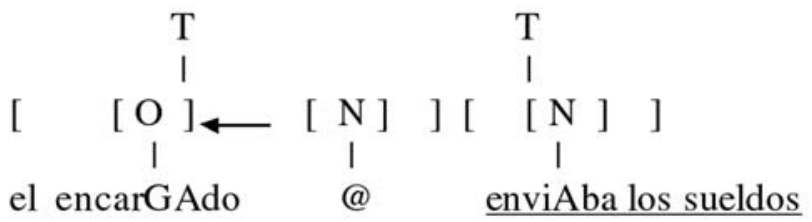

\subsection{Final focus}

The structure proposed for this intonation contour by Cabrera Abreu, García Lecumberri, Maidment and Takahashi (1999) was introduced in $\S 4.4,(11)$ above. There we reported that the authors accounted for the non-focal stretch of the intonation contour in terms of two intonation groups in which an empty nucleus was licensed by virtue of the final-empty-nucleus parameter, and that the empty nuclei themselves licensed an onset by virtue of onset licensing. Notice, however, that this structure predicts a downstep effect between the first two intonation groups, something which we do not observe in our data. In fact, in the description presented in $\$ 2.4$ above, although there is a drop in pitch, the crucial point is that the 
second peak is higher than the first one, but there is no perceived downstep effect. The observed drop in pitch can be accounted for by toneless boundaries which do not necessarily have to correspond to an empty nucleus.

The reason which lead Cabrera Abreu, García Lecumberri, Maidment, and Takahashi (1999) to put forward this proposal is that it allows them to draw the generalisation that pre-focal structures for medial and final focus utterances typically show this constituent structure, and therefore, yield extremely similar prefocal rising movements.

However, in this paper, we propose an alternative well-formed phonological representation which does not predict the unobserved downstep effect, and which therefore conforms to descriptive adequacy. We illustrate such a representation in (16) below.

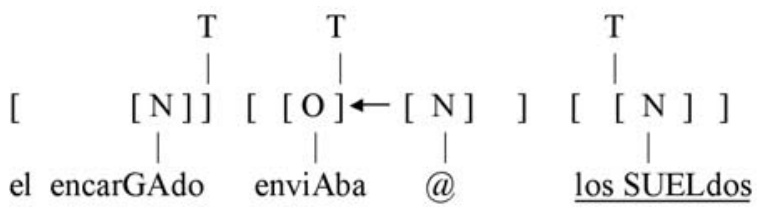

The prefocal item el encarGAdo is licensed by a nucleus which is obligatory in this intonation group, and is aligned with its rightmost edge. T associated to the rightmost boundary of the intonation group is responsible for the rising movement over el encarGAdo. In the next intonation group, an empty nucleus is licensed by virtue of the final empty nucleus parameter. Such a nucleus is responsible for the observed downstep effect taking place before the last peak. In addition, the empty nucleus licenses the onset by onset licensing, and this is aligned with the left edge of the intonation group. $\mathrm{T}$ associated to the right boundary of the onset accounts for the rising movement over enviAba. The focal item, $\underline{\text { SUELdos }}$ is licensed by a nucleus, whose left boundary shows $\mathrm{T}$ associated. This is responsible for the observed falling movement in the F0 contour.

Although we favour this analysis - since it conforms to the principles of licensing presented thus far, and also achieves descriptive adequacy -, it should also be taken cautiously until further research into the principles governing phonological structure is performed, and more data is analysed. The reason is that, even though there is a win in descriptive adequacy, there is also a loss in phonological generalisation. Notice that, the structure in (16) may lead us to assume that the two rising movements over the prefocal items are different since they show different constituent structures. The first one is licensed by a nucleus, whereas the second one shows an onset / empty nucleus structure. As far as our data is concerned, there is no evidence which may allow us to either confirm or reject this assumption. In addition, (16) may be wrongly understood as showing two focal items, since there are two constituents licensed by a nucleus. This is also undesirable, and needs further study. 


\section{Conclusion}

In this paper, we have proposed an account of Spanish intonational focus in terms of constituents such as onsets, nuclei and intonation groups integrated in phonological structure by means of licensing principles which are also responsible for the association of $\mathrm{T}$ to their constituent-boundaries.

The typical phonological features manifested by the focal item are the following: (a) it is licensed by an obligatory nucleus, which is aligned with the right edge of the intonation group; (b) T associated to the leftmost boundary of the nucleus accounts for the observed falling movement; and (c) the presence of a toneless nuclear constituent in a preceding intonation group is responsible for the also observed downstep effect on the focal item.

Concerning the prefocal item (a) it tends to be licensed by an optional onset which is licensed by an empty nucleus, which is itself subsequently licensed by the right edge of the intonation group; (b) $\mathrm{T}$ associated to the rightmost boundary of the onset is responsible for the typical rising movement over this item.

There are certainly a few issues which remain unsolved, like, for instance, the representation for those intonation contours in which there seems to be more than one constituent in the prefocal material. We may wonder which is the phonological structure which best accounts for the rising movements, bearing in mind that the proposal of a nuclear constituent in this position may lead on to the undesirable assumption that such a constituent licenses focal material.

Another matter which requires further study and whose implications may go beyond the account of Spanish intonational focus is the interpretation of a phonological structure which shows an intonation group containing an onset followed by an empty nucleus, with no further intonation groups following. Presumably, the downstep effect would apply vacuously since there are no following constituents whose boundaries can show $\mathrm{T}$ associated to, and which can undergo the aforementioned effect. If this is the case then, what is the purpose of an empty nucleus in the first place? This remains unsolved until a detailed analysis of the phonological principles governing constituent structure are tested against further data from other languages.

\section{References}

Cabrera Abreu, Mercedes (1999). «Phonological analysis of medial focal accent in Spanish and English». Proceedings of the XXII AEDEAN Conference. Lleida.

- (2000). A Phonological Model for Intonation Without Low Tone. Indiana: Indiana University Linguistics Club Publications.

Cabrera Abreu, Mercedes; García Lecumberri, María Luisa; Maidment, John; Takahashi, Toyomi (1999). «The phonological representation of accentual focus in some English and Spanish utterances». Proceedings of the $14^{\text {th }}$ International Congress of Phonetic Sciences, San Francisco, pp. 1761-1764.

Estebas-Vilaplana, Eva (2000). The Use and Realisation of Accentual Focus in Central Catalan with a Comparison to English. University College London, doctoral dissertation. 
Face, Timothy (2002). Intonational Marking of Contrastive Focus in Madrid Spanish. Munich: Lincom Europa.

Frota, Sónia (2002). «The prosody of focus: a case-study with cross-linguistic implications». In: Bel, Bernard; Marlin, Isabelle (eds.). Proceedings of the Speech Prosody 2002 Conference, Aix-en-Provence: Laboratoire Parole et Langage, pp. 319-322.

García Lecumberri, María Luisa (1995). Intonational Signalling of Information Structure in English and Spanish: a Comparative Study. Bilbao: Servicio Editorial Universidad del País Vasco.

García Lecumberri, María Luisa; Cabrera Abreu, Mercedes (1999). «Dominio del foco acentual en español frente al inglés: cuestiones de ámbito y ambigüedad». Actas del VI Simposio Internacional de Comunicación Social, Santiago de Cuba, pp. 185-190.

García Lecumberri, María Luisa; Cabrera Abreu, Mercedes; Maidment, John (1997). «The realisation of focal accent in English and Spanish: similarities and differences». Proceedings of an ESCA Workshop. Intonation: Theory, Models and Applications, Athens, pp. 141-143.

Harris, John (1994). Sound Structure of English. Oxford: Blackwell.

Hualde, José Ignacio (2002). «Intonation in Spanish and the other Ibero-Romance languages: overview and status quaestionis». In: Wiltshie, Caroline; Camps, Joaquim (eds.). Romance phonology and variation. Amsterdam: Benjamins, pp. 37-69.

Kaye, Jonathan; Lowenstamm, Jean; Vergnaud, Jean-Roger (1990). «Constituent structure and government in phonology». Phonology 7, 2: 193-232.

Ladd, D. Robert (1996). Intonational Phonology. Cambridge: Cambridge University Press.

Nibert, Holly (2000). Phonetic and Phonological Evidence for Intermediate Phrasing in Spanish Intonation. University of Illinois at Urbana-Champaign, doctoral dissertation.

O'Connor, JD.; Arnold, GF (1973). Intonation of Colloquial English. London: Longman.

Pierrehumbert, Janet B. (1987). The Phonetics and Phonology of English Intonation. Indiana: Indiana University Linguistics Club.

Prieto, Pilar (1995). «Aproximació als contorns tonals del català central». Caplletra 19: 161-186.

- (1998). «The scaling of the L values in Spanish downstepping contours». Journal of Phonetics 26: 261-282.

- (2002). Review of Sosa, Juan Manuel (1999). La entonación del español. Madrid: Cátedra, pp. 264. Linguistics 39: 1192-1201. 